\title{
Isotopes of the Alkaline Earth Metals from Uranium
}

$\mathrm{D}^{\mathrm{i}}$ URING the last two years, the bombardment of uranium by neutrons has led to some striking results, due primarily to the combined efforts of Prof. O. Hahn, L. Meitner and F. Strassmann, working in the Kaiser Wilhelm Institute of Chemistry in Berlin-Dahlem. In addition to three artificial isotopes of uranium, they have succeeded in establishing the existence of six trans-uranium elements of atomic numbers 93-96, and the existence of a seventh trans-uranium element (half-value period $=60$ days) is indicated.

In similar experiments, I. Curie and P. Savitch obtained evidence of the production of a substance with a half-value period of $3 \frac{1}{2}$ hours. The identity of this substance is uncertain, and although the authors have considered several possibilities, none of them is very satisfactory. Recent experiments on the chemical properties of the trans-uranium elements have led O. Hahn and F. Strassmann (Naturwissenschaften, 26, 755, Nov. 18, 1938 ; and 27, 11, Jan. 6, 1939) to look for this substance (half-value period = $3 \frac{1}{2}$ hours), and they have been successful in obtaining it by the same methods as those used by the French workers. Their investigations have led to remarkable results which are summarized in what follows.

The bombardment of uranium with neutrons apparently resulted in the production of four isomeric isotopes of 'radium', which must have arisen by two successive $\alpha$-ray transformations via thorium. These four 'radium' isotopes emit $\beta$-rays and yield four isomeric isotopes of 'actinium', which, in their turn, by further emission of $\beta$-rays, presumably give rise to four isotopes of 'thorium', but details of these last products are still incompletely known. The results so far obtained may be represented provisionally by the following scheme, in which the nature of the radiations emitted and the revised half-value periods of the new products are indicated.

Should this scheme adequately represent the facts, these twelve new substances should have atomic weights of value 231, and since a natural thorium isotope (UY) of atomic weight 231 and half-value period 25 hours is already known, it will be of interest to investigate whether one of the four 'thorium' isotopes is identical with UY.

Hahn and Strassmann believe that the substance found by Curie and Savitch is a mixture of the abovementioned isotopes, each of which has been individually detected and chemically examined. Moreover, the properties of such a mixture would agree with those of the substance detected by the latter authors, who suspect that their substance also contains others of longer life, but of unknown genesis.
An interesting feature of this work is that the production of the new isotopes is enhanced by the use of slow neutrons, and the $\alpha$-particle disintegrations indicated in the earlier stages of the process are believed to be the first instances of $\alpha$-particle emission effected by slow neutrons. It is, of course, possible that the quadruplicity of isotopes resulting from the bombardment of uranium by neutrons takes place in ${ }_{92}^{239} \mathrm{U}$, as in the case of the trans-uranium elements, and the intermediate 'thorium' isotope ${ }_{90}^{235} \mathrm{Th}$ may also possess four half-value periods.

In the second of their publications mentioned above, Hahn and Strassmann give particulars of the chemical methods employed in establishing their results, as well as a number of activity curves from which some of the half-value periods have been derived. In order to establish beyond question the chemical nature of the products they have designated 'radium' isotopes, which were separated with barium, the authors carried out fractional erystallizations and precipitations with the active barium salts, by the method used for altering the concentration of radium in salts of barium containing radium.

As is well known, crystals richer in radium can be obtained by fractional crystallization of the chloride, bromide or chromate of barium, when it contains radium. In experiments on these lines, carried out by Hahn and Strassmann with their active barium preparations from which disintegration products had been removed, negative results were always obtained, that is, the activity was uniformly distributed amongst the various barium fractions, whereas control experiments with barium containing the radium isotopes $\mathrm{ThX}$ and $\mathrm{Ms}^{\mathrm{T}} \mathrm{Th}_{1}$ yielded results conforming to those obtained when the barium contains ordinary radium. In a further experiment, in which 'Raiv' and MsTh $_{1}$ were mixed with barium and then subjected to fractional crystallization, only the $\mathrm{MsTh}_{1}$ was concentrated in the process. The authors conclude from these results that their 'radium' isotopes have the properties of barium, and that, from the chemical point of view, other elements being excluded, these active substances must be re. garded as consisting not of radium but of barium. This conclusion has been confirmed by further indicator experiments, according to a personal communication from one of the authors (O.H.).

Hahn and Strassmann have also subjected a mixture of their ' $A c_{i i}$ ' and the actinium isotope $\mathbf{M s T h}_{2}$ with lanthanum oxalate to fractionation from a nitric acid solution. At the end of the process a gain in concentration of $\mathrm{MsTh}_{2}$ was achieved, but no increase in the concentration of ' $A c_{i i}$ ' was noted. From this they conclude that the substances generated by $\beta$-ray emission from their alkaline-earth products are not isotopes of actinium, a conclusion which agrees with that of Curie and Savitch for their composite product of half-value period $3 \frac{1}{2}$ hours. In all probability they will prove to be isotopes of lanthanum. The products designated 'Th' in the above scheme of disintegration, which constitute the final members of the series 
derived from the 'Ac-La' preparations, have not yet been tested for their identity with cerium.

On the strength of the chemical evidence, it is difficult to avoid the conclusion that the elements denoted by the symbol ' $R \mathrm{a}$ ' in the above-mentioned disintegration scheme should in reality be designated 'Ba', and so far as the evidence has gone it seems probable that the succeeding elements 'Ac' and ' $T h$ ' will prove to be 'La' and 'Ce' respectively. It is as though, at some stage in the process resulting from the bombardment of uranium by neutrons, a 'collapse' of the nucleus occurs, giving rise to the four active isomeric alkaline-earth elements and the succeeding 'lanthanum' and 'cerium' products. The investigations are being continued.
R. W. L.

\section{Subspecies and Varieties}

\begin{abstract}
A DISCUSSION dealing with subspecies and varieties was held at the meeting of the Linnean Society on February 2. This discussion had been arranged at the request of the Association for the Study of Systematies in Relation to General Biology in order to obtain information as to the views and principles governing the practice of systematists in various groups of animals and plants. It was apparent from the diseussion that systematists are generally dissatisfied with the existing state of affairs. As was to be expected, little or no attention is paid to infra-specific categories in the less-known groups of both plant and animal kingdoms; but where the broader taxonomic outlines are well understood there is considerable divergence in systematic practices according to the amount of genetical, cytological and ecological work that has been done. In vertebrates, only a single infra-specific category, the 'subspecies', is generally recognized, but in entomology and botany there are others. How many there may be and their status in the taxonomic scheme are matters of dispute, and there is a disturbing confusion in the terminology applied to them. In this connexion, it may be mentioned that the Association is compiling a list of the various terms which have been used. Almost all the speakers stressed the need for more experimental work from the genetical, cytological, physiological and ecological aspects.
\end{abstract}

In opening the discussion, Mr. M. A. C. Hinton described the practice of mammalogists, who, in recent years, have used the term 'subspecies' to express stages or trends in geographical variation; they are sections of what Dr. Julian Huxley has called 'clines' (Nature, 142, 219; 1938). One principle generally accepted is that no two races of the same species can ever be found on the same ground, though exceptionally two such forms may meet after very different histories and journeys and continue to exist side by side without fusing. The principle that, unless intergradation can be demonstrated, differences, however trivial, indicate specific separation, has some theoretical support. In practice, however, it has disadvantages, especially in dealing with insular forms, and tends to mask that most interesting and instructive phenomenon, discontinuous distribution.

The definition of subspecies is an essential part of any intensive analysis of the facts of variation and distribution, and is particularly valuable in palæontology, by preventing loose identifications and consequent faulty geological deductions. It is hoped soon to commence large-scale experiments to test the values and permanence of subspecific characters and to obtain more definite information on subjects concerning which surmise alone is at present possible.
Dr. W. B. Turrill emphasized that all taxonomic categories are matters of scientific convenience based on abstractions from a continuous evolution. He recognizes that for little-known floras it is undesirable to analyse below the species level; but urged that in relatively well-studied floras the need is for intensive studies of infra-specific variation from every angle. His own researches have led him to the conclusion that taxonomic categories intergrade completely. He suggested that the term 'variety' should be used for every phenotype which is the expression of a different genotype, and that the term subspecies should be used only when a species is in process of breaking up into new ones. Names should be given to infra-specific groups only to serve some definite purpose, and in highly polymorphic species symbols might be used instead. Apomicts should receive distinct treatment.

Mr. A. J. Wilmott pointed out that confusion results from using the words species, subspecies and variety both for eategories of variation in Nature and groups of different rank in the nomenclatural system. He suggested that the terms 'binome' and 'trinome' might be substituted for species and subspecies in the nomenclatural sense, leaving the latter free for application to categories of variation. True subspecies are parts of the present time section of a lineage which has become branched by isolation, but the branches of which arenot yet completely separated. Varieties, on the other hand, are in a different category, being merely the observed phenotypic effects of separate genes, and not different kinds of organisms; they should not be given names under the same system as species and subspecies, but might be designated by symbols.

Mr. J. S. L. Gilmour dealt with the philosophical aspect of the subject and drew a distinction between taxonomic and non-taxonomic infra-specific categories. The former, which might be limited to subspecies, variety, and form, should be based so far as possible on the total attributes of the individuals concerned, while the latter should be based on a selection of attributes chosen for special purposes. Examples of such non-taxonomic categories are Dancer's 'commiscuum','comparium', and 'convivium', based on interfertility data, and useful for investigating the relationship between such data and other attributes, such as morphological differentiation. He urged that this distinetion is essential for the proper classification of infra-specific variability.

Mr.H.W.Parkershowed that the practice of herpetologists is essentially similar to that of mammalogists. $\mathrm{He}$ believes, however, that owing to lack of other data, systematists have laid undue emphasis on geographical considerations, with invidious results. Recognition by trinomials of certain forms to the 